\title{
Silicon Grignard Reagents as Nucleophiles in Transition-Metal- Catalyzed Allylic Substitution
}

\section{Weichao Xue \\ Martin Oestreich*}

Institut für Chemie, Technische Universität Berlin, Strasse des 17. Juni 115, 10623 Berlin, Germany

martin.oestreich@tu-berlin.de

Published as part of the 50 Years SYNTHESIS Golden Anniversary Issue

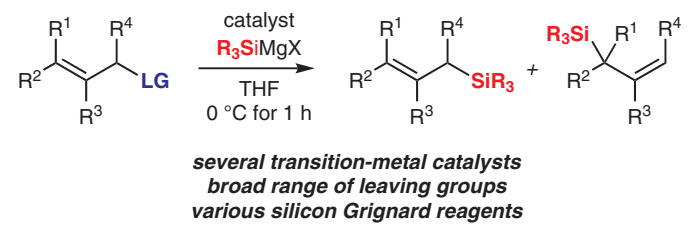

Copper-catalyzed allylic substitution with $R_{3} \operatorname{SiBpin}$ or $\left(R_{3} S i\right)_{2} Z n$

Received: 24.09.2018

Accepted: 28.09.2018

Published online: 22.10 .2018

DOI: 10.1055/s-0037-1610309; Art ID: ss-2018-z0648-fa

License terms: cc)

Abstract A broad range of transition-metal catalysts is shown to promote allylic substitution reactions of allylic electrophiles with silicon Grignard reagents. The procedure was further elaborated for Cul as catalyst. The regioselectively is independent of the leaving group for primary allylic precursors, favoring $\alpha$ over $\gamma$. The stereochemical course of this allylic transposition was probed with a cyclic system, and anti-diastereoselectivity was obtained.

Key words allylic substitution, copper, Grignard reagents, silicon

Allylic silanes are an often-used class of silicon reagents and continue to be widely applied in synthesis. ${ }^{1}$ Several methods are available that provide reliable access to these compounds. ${ }^{2-6}$ One established methodology is by transition-metal-catalyzed allylic substitution of allylic precursors with silicon (pro)nucleophiles such as $\mathrm{Si}-\mathrm{Si}^{2}$ and $\mathrm{Si}-\mathrm{B}^{3}$ compounds as well as zinc ${ }^{4}$ reagents. Examples with copper complexes as catalysts pertinent to the present study are summarized in Scheme 1 (top). The reverse approach, that is, the nucleophilic displacement at silicon electrophiles with carbon nucleophilic, is far less general. ${ }^{5}$

We recently developed a robust method for the preparation of bench-stable solutions of silicon Grignard reagents 1 (Scheme 1, bottom). ${ }^{7}$ These had essentially been not available previously, ${ }^{8}$ and we decided to assess their suitability as silicon nucleophiles in allylic substitution reactions, particularly with emphasis on the influence of the leaving group on the regioselectivity. Herein, we describe the application of silicon Grignard reagents to allylic substitution reactions catalyzed by manganese, iron, cobalt, nickel, copper, and palladium salts.

We started our investigation by exploring the coupling reaction of commercially available $E$-cinnamyl acetate $[(E)$ 2a] and $\mathrm{Me}_{2} \mathrm{PhSiMgX} \mathbf{1 a}$ (Table 1). At the beginning, several first-row metal salts were employed as catalysts ( $5 \mathrm{~mol} \%$ )

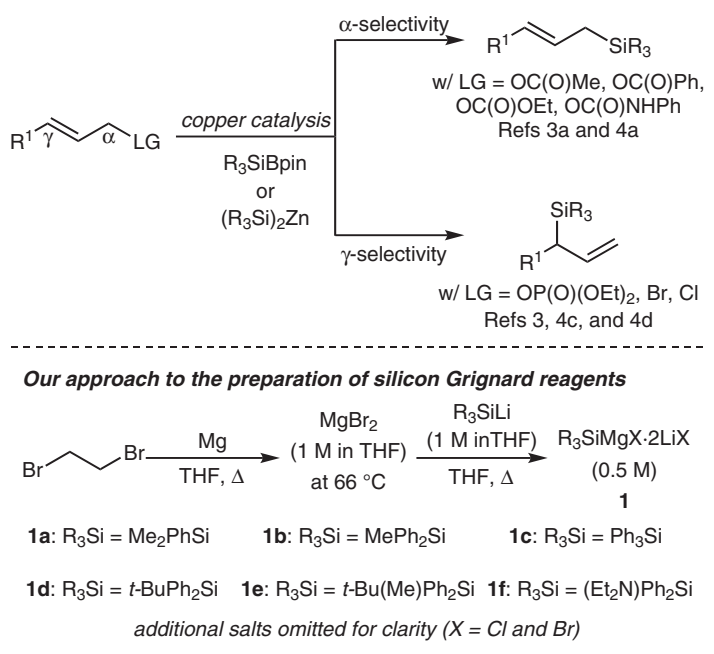

Scheme 1 Copper-catalyzed allylic substitution with silicon (pro)nucleophiles (top; LG: leaving group) and preparation of silicon Grignard reagents (bottom)

without additional ligands (Table 1, entries 1-6). Any of these catalysts enabled the reaction, affording the linear allylic silane $\alpha-(E)$-3a in near-quantitative yields using $\mathrm{NiBr}_{2}$.glyme, CuI, and $\mathrm{CuCN}$; however, $\mathrm{MnBr}_{2}, \mathrm{FeCl}_{3}$, and $\mathrm{CoCl}_{2}$ furnished the desired product in somewhat lower yields. Also, (E)-2a underwent silylation in the presence of $\mathrm{PdCl}_{2}$ (entry 7). In all these reactions, the thermodynamically favored $\alpha$-regioisomer was formed with high $\alpha / \gamma$ ratio. The yield remained high when $2 \mathrm{~mol} \%$ of CuI were employed. A blank experiment without catalyst gave no conversion (entry 8).

With the ligand-free, copper-catalyzed procedure in hand, we probed the effect of various leaving groups $[(E)$ $\mathbf{2 a}-\mathbf{i} \rightarrow \alpha-(E)-3 \mathbf{a}$ and $\gamma-\mathbf{3 a}$, Table 2]. Next to model substrate (E)-2a, E-cinnamyl alcohols activated as carboxylate [as in $(E)-\mathbf{2 b}$ ], carbonates [as in $(E)-\mathbf{2 c}$ and $(E)-\mathbf{2 d}$ ], carbamate [as in $(E)-\mathbf{2 e}$ ], and phosphate [as in $(E)-\mathbf{2 f}$ ] participated well in 
Table 1 Selected Examples of the Catalyst Screening ${ }^{a}$

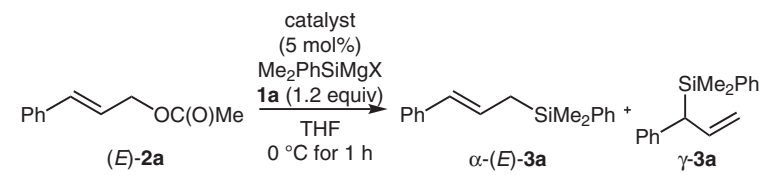

\begin{tabular}{lllll}
\hline Entry & Catalyst & $E / Z$ of $\alpha-3 a^{\mathrm{b}}$ & $\alpha / \mathrm{Y}^{\mathrm{b}}$ & Yield $(\%)^{\mathrm{b}}$ of 3a \\
\hline 1 & $\mathrm{MnBr}_{2}$ & $99: 1$ & $96: 4$ & 67 \\
2 & $\mathrm{FeCl}_{3}$ & $99: 1$ & $98: 2$ & 79 \\
3 & $\mathrm{CoCl}_{2}$ & $99: 1$ & $99: 1$ & 81 \\
4 & $\mathrm{NiBr}_{2} \cdot$ glyme & $99: 1$ & $98: 2$ & 94 \\
5 & $\mathrm{Cul}$ & $99: 1$ & $99: 1$ & $95(95)^{\mathrm{c}}$ \\
6 & $\mathrm{CuCN}$ & $99: 1$ & $99: 1$ & 95 \\
7 & $\mathrm{PdCl}_{2}$ & $97: 3$ & $95: 5$ & 80 \\
8 & none & - & - & trace \\
\hline
\end{tabular}

a Reactions performed on a $0.50 \mathrm{mmol}$ scale.

${ }^{b}$ Yield is for the mixture of isomers and was determined by GLC analysis with tetracosane as an internal standard.

' With Cul (2 mol\%).

this silylation (Table 2, entries 1-6); yields were generally high and $\alpha / \gamma$ ratios and $E / Z$ selectivities were good. Cinnamyl halides $(E)-\mathbf{2 g}$ and $(E)-\mathbf{2 h}$ were also included into the survey (entries 7 and 8 ), again leading to high yields but to slightly diminished regioselectivities. This outcome, that is $\alpha$-selectivity for all tested leaving groups, stands in stark contrast to earlier findings in copper-catalyzed allylic substitution with $\mathrm{Si}-\mathrm{B}$ compounds ${ }^{3}$ and silicon zinc reagents ${ }^{4}$ (see Scheme 1, top). As expected, the allylic substitution did not occur with free cinnamyl alcohol [(E)-2i] (entry 9).
Table 2 Investigation of Leaving Groups ${ }^{\mathrm{a}}$

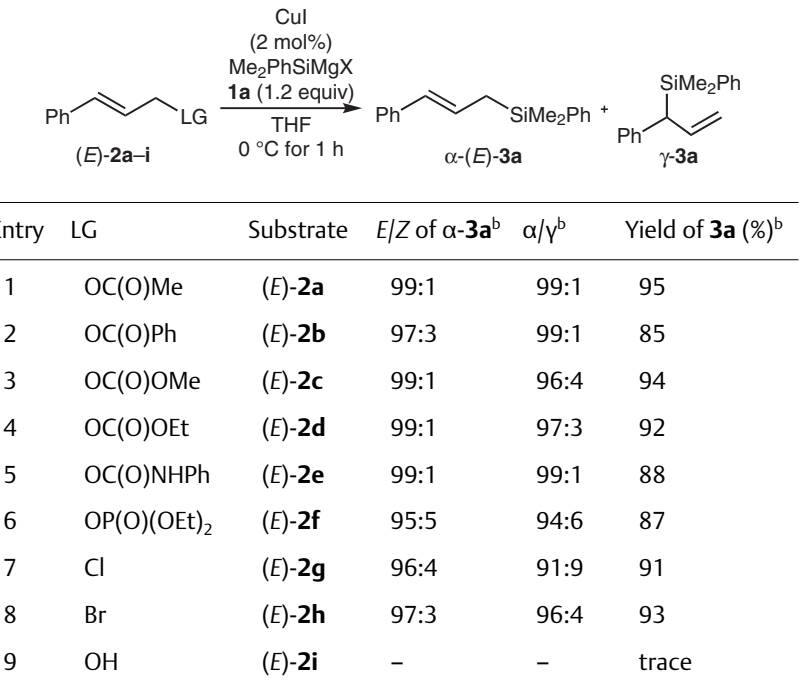

a Reactions performed on a $0.50 \mathrm{mmol}$ scale.

${ }^{b}$ Yield is for the mixture of isomers and was determined by GLC analysis with tetracosane as an internal standard.

This allylic substitution was then applied to a variety of primary allylic precursors using $\mathrm{Me}_{2} \mathrm{PhSiMgX} \mathbf{1 a}$ (Scheme 2 ). In accordance with the previous observations (Tables 1 and 2), isomerically pure geranyl acetate $(E)-\mathbf{4 a}$ and neryl acetate $(Z)-\mathbf{4 a}$ reacted cleanly to produce allylic silanes $\alpha$ $(E)-8 \mathbf{a}$ and $\alpha-(Z)-\mathbf{8 a}$, respectively, with exclusive preservation of the double bond geometry and excellent $\alpha / \gamma$ selectivity. Allylic bromide $(E)-5 \mathbf{h}$ underwent silylation equally well, however, with reduced regio- and diastereoselectivi-

\section{Biographical Sketches}
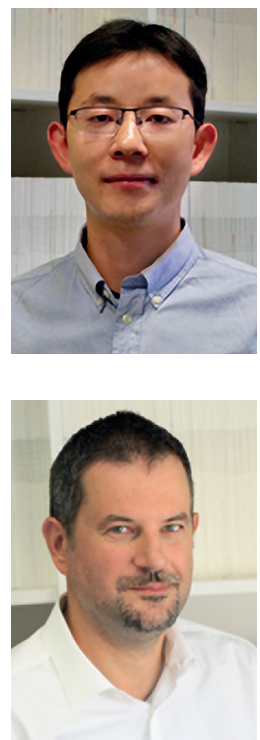

Weichao Xue (born in 1989 in Pingdingshan/China) studied Chemistry at Henan University (2008-2012) and Shanghai University (2012-2015). He obtained his bachelor's degree with Feng Shi (Kaifeng, 2012) and master's degree with Hegui

Martin Oestreich (born in 1971 in Pforzheim/Germany) is Professor of Organic Chemistry at the Technische Universität Berlin. He received his diploma degree with Paul Knochel (Marburg, 1996) and his doctoral degree with Dieter Hoppe
Gong (Shanghai, 2015). He then moved to Berlin to pursue docChina Scholarship Council (2015-2019). Currently, he is a Ph.D. candidate in the group of Martin Oestreich at the Technische Universität Berlin. $\mathrm{He}$ is toral research funded by the

also a member of the Berlin Graduate School of Natural Sciences and Engineering (BIGNSE) of the Cluster of Excellence Unifying Concepts in Catalysis of the Deutsche Forschungsgemeinschaft.

(Münster, 1999). After a twoyear postdoctoral stint with Larry E. Overman (Irvine, 19992001), he completed his habilitation with Reinhard Brückner (Freiburg, 2001-2005) and was appointed as Professor of Organic Chemistry at the West- fälische Wilhelms-Universität Münster (2006-2011). He also held visiting positions at Cardiff University in Wales (2005), The Australian National University in Canberra (2010), and Kyoto University (2018). 
ties. As expected, simple primary allylic electrophiles such as $\mathbf{6 a}$ and $\mathbf{7 h}$ were converted into corresponding silylated products in good yields.

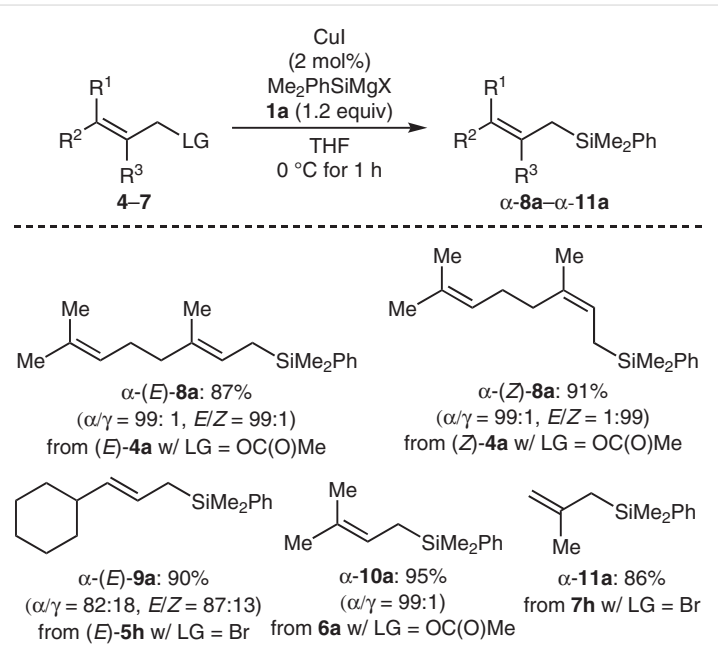

Scheme 2 Copper-catalyzed allylic substitution of primary allylic precursors with silicon Grignard reagents. Yields are for the mixture of isomers, and regiochemical and diasteromeric ratios were confirmed by ${ }^{1} \mathrm{H}$ NMR analysis.

Unlike primary allylic sources that engage in an $S_{N}$ pathway with high regiocontrol, the regiochemical situation is different for secondary substrates. Cyclic 13a was obtained in high yield starting from the secondary bromide $\mathbf{1 2 h}$ (Scheme 3, eq 1). Acyclic 14b was transformed into $\gamma-(Z)-$ 15a with excellent $\gamma$-selectivity, corresponding to an $S_{N}{ }^{\prime}$ mechanism (Scheme 3, eq 2). Interestingly, the $Z$-isomer

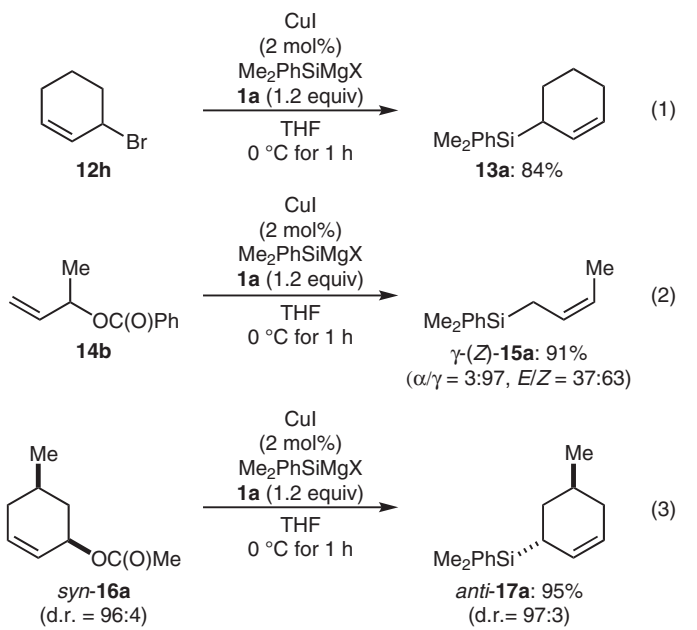

Scheme 3 Copper-catalyzed allylic substitution of secondary allylic precursors with silicon Grignard reagents. Yields are for the mixture of isomers, and regiochemical and diasteromeric ratios were confirmed by ${ }^{1} \mathrm{H}$ NMR analysis. was formed predominantly, which is different from literature precedence. ${ }^{4 \mathrm{a}, 9}$ To further distinguish between anti- $\mathrm{S}_{\mathrm{N}}{ }^{\prime}$ and $s y n-S_{\mathrm{N}}{ }^{\prime}$ mechanisms, cyclic allylic carboxylate syn-16a was synthesized and subjected to the standard condition (Scheme 3, eq 3). ${ }^{10}$ Indeed, syn-16a was converted into anti-17a with complete inversion of the stereochemical information. This result is consistent with related copperpromoted allylic substitutions. ${ }^{3 \mathrm{f}, 4 \mathrm{a}, 11}$

Continuing with allyl methyl carbonate (18c), different silicon Grignard reagents $\mathbf{1}$ were subjected to the standard setup (Scheme 4). Similar to $\mathrm{Me}_{2} \mathrm{PhSiMgX} \mathrm{1a,} \mathrm{yields} \mathrm{are}$ generally excellent for regularly used $\mathrm{MePh}_{2} \mathrm{Si}$ (from 1b) and $\mathrm{Ph}_{3} \mathrm{Si}$ (from 1c) as well as more hindered $t$-BuPh${ }_{2} \mathrm{Si}$ (from 1d) and $t$-Bu(Me)PhSi (from 1e). The same result was obtained with heteroatom-substituted silicon nucleophile 1f, containing Tamao's silicon anion. ${ }^{12}$

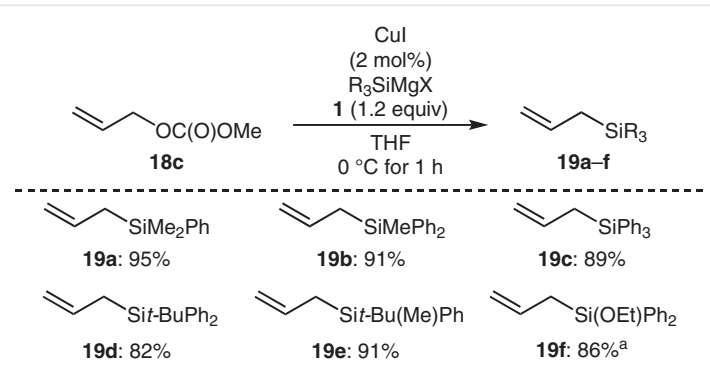

Scheme 4 Copper-catalyzed allylic substitution of allylic precursor $\mathbf{1 8 c}$ with silicon Grignard reagents. Yields are for the mixture of isomers, and ratios were determined by ${ }^{1} \mathrm{H}$ NMR analysis. ${ }^{\mathrm{a}} \mathrm{EtOH} / \mathrm{NH}_{4} \mathrm{Cl}$ added after reaction.

Considering the challenges associated with the construction of silicon-stereogenic silanes, ${ }^{13}$ we attempted an enantioselective version of this allylic substitution in the presence of chiral ligands (Scheme 5). The reaction of racemic $t$-Bu(Me)PhSiMgX 1e and allylic precursor 18c was chosen as a model reaction. Several catalytic systems were tested but neither led to the asymmetric induction at the silicon atom.

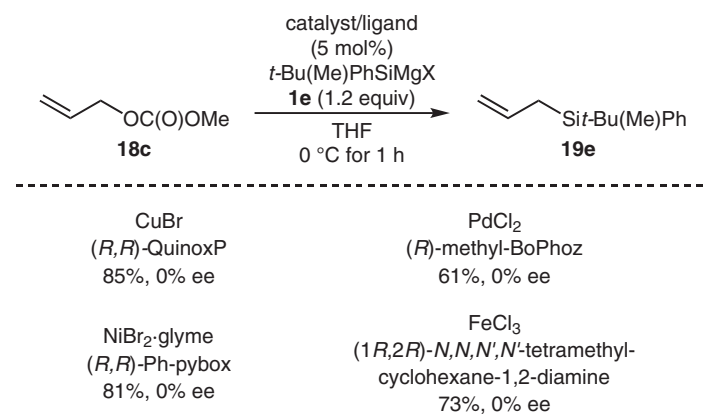

Scheme 5 Attempted enantioselective allylic substitution of allylic carbonate $\mathbf{1 8 c}$ with $t$-Bu(Me) PhSiMgX (1e) 
To summarize, we have disclosed here a practical method for the synthesis of allylic silanes from readily accessible allylic precursors and easy-to-handle silicon Grignard reagents. Several metal salts can promote this transformation in moderate to excellent yields without the need of added ligand. The leaving-group scope is broad, comprising the usual oxygen leaving groups as well as halides.

All reactions were performed in flame-dried glassware using conventional Schlenk techniques under a static pressure of $\mathrm{N}_{2}$, unless otherwise stated. Liquids and solutions were transferred with syringes. $\mathrm{CuI}$ (anhyd CuI, 99\%, ABCR), other metal salts, and chiral ligands were purchased from commercial suppliers and used as received. Allylic precursors 2a, 2g, 2h, 2i, (E)-4a, (Z)-4a, 6a, 7h, 12h, and 18c are commercially available. Compounds $\mathbf{2 b},{ }^{4 \mathrm{c}} \mathbf{2 c},{ }^{4 \mathrm{c}} \mathbf{2 e},{ }^{4 \mathrm{c}} \mathbf{2 f},{ }^{4 \mathrm{c}}(E)-\mathbf{5 h},{ }^{4 \mathrm{c}} \mathbf{1 4 b},{ }^{14}$

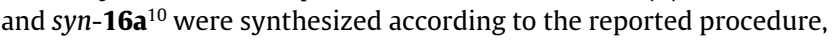
and all spectroscopic data matched those reported. THF was dried over $\mathrm{Na}$ or $\mathrm{K} /$ benzophenone and distilled prior to use. Technical grade solvents for extraction or chromatography (cyclohexane, $\mathrm{CH}_{2} \mathrm{Cl}_{2}$, EtOAc, and $n$-pentane) were distilled prior to use. Analytical TLC was performed on silica gel 60 F254 glass plates from Merck. Flash column chromatography was performed on silica gel $60(40-63 \mu \mathrm{m}, 230-400$ mesh, ASTM) from Grace using the indicated solvents. ${ }^{1} \mathrm{H},{ }^{13} \mathrm{C}$, and ${ }^{29} \mathrm{Si}$ DEPT NMR spectra were recorded in $\mathrm{CDCl}_{3}$ on Bruker AV400 and AV500 instruments. Chemical shifts are reported in parts per million (ppm) and are referenced to the residual solvent resonance as the internal standard $\left(\mathrm{CHCl}_{3}: \delta=7.26\right.$ for ${ }^{1} \mathrm{H} \mathrm{NMR}$ and $\mathrm{CDCl}_{3}: \delta=77.0$ for ${ }^{13} \mathrm{C}$ NMR). ${ }^{29} \mathrm{Si}$ is referenced in compliance with the unified scale for NMR chemical shifts as recommended by the IUPAC stating the chemical shift relative to $\mathrm{BF}_{3} \cdot \mathrm{Et}_{2} \mathrm{O}, \mathrm{CCl}_{3} \mathrm{~F}$ and $\mathrm{Me}_{4} \mathrm{Si}^{15}$ Data are reported as follows: chemical shift, multiplicity (standard abbreviations), coupling constant $(\mathrm{Hz})$, and integration. Gas liquid chromatography (GLC) was performed on a Varian 430-GC gas chromatograph equipped with a Varian FactorFour Capillary column $(30 \mathrm{~m} \times 0.25 \mathrm{~mm}, 0.25 \mu \mathrm{m}$ film thickness). Enantiomeric excesses were determined by analytical high-performance liquid chromatography (HPLC) analysis on an Agilent Technologies 1290 Infinity instrument with a chiral stationary phase using Daicel Chiralcel OJ-RH, ( $\mathrm{MeCN} / \mathrm{H}_{2} \mathrm{O}$ mixtures as solvent). Melting points were determined using a Leica Galen III melting point apparatus. Mass spectra (MS) were obtained from the Analytical Facility at the Institut für Chemie, Technische Universität Berlin.

\section{Preparation of $\mathbf{R}_{\mathbf{3}} \mathrm{SiMgX} \mathbf{1}$; General Procedure 1 (GP 1 )}

At $0{ }^{\circ} \mathrm{C}$, the required chlorosilane $(24.0 \mathrm{mmol}, 1.0$ equiv) was added to a flame-dried Schlenk flask charged with activated Li chunks (666 $\mathrm{mg}, 96.0 \mathrm{mmol}, 4.0$ equiv) suspended in THF $(20 \mathrm{~mL})$, and the resulting suspension was stirred at this temperature overnight under $\mathrm{N}_{2}$ atmosphere to give $\mathrm{R}_{3} \mathrm{SiLi}$. The concentration of $\mathrm{R}_{3} \mathrm{SiLi}(\sim 1.0 \mathrm{M}$ in THF, approximately $80-90 \%$ conversion) was determined by titration against diphenylacetic acid (Kofron's method). ${ }^{16} \mathrm{~A}$ flame-dried twonecked round-bottomed flask charged with a magnetic stir bar and equipped with a water condenser is connected to a Schlenk line and purged with $\mathrm{N}_{2}$. The flask was charged with $\mathrm{Mg}$ turnings (292 mg, $12.0 \mathrm{mmol}, 1.2$ equiv) followed by the addition of THF $(10 \mathrm{~mL})$ and was then heated to $66^{\circ} \mathrm{C}$. 1,2-Dibromoethane ( $1.88 \mathrm{~g}, 10.0 \mathrm{mmol}, 1.0$ equiv) was quickly added via syringe, and the reaction mixture was heated at reflux for $3 \mathrm{~h}$ at high water-flow rate to afford $\mathrm{MgBr}_{2}(1.0 \mathrm{M}$ in THF at $\left.66{ }^{\circ} \mathrm{C}\right)$. Then, the corresponding $\mathrm{R}_{3} \mathrm{SiLi}$ solution $(10 \mathrm{mmol}$, 1.0 equiv) was subsequently added dropwise to the $\mathrm{MgBr}_{2}$ solution over $10 \mathrm{~min}$ at this temperature. $\mathrm{R}_{3} \mathrm{SiMgX} \cdot 2 \mathrm{LiX}$ solution formed was cooled to r.t. The concentration of $\mathrm{R}_{3} \operatorname{SiMgX} \cdot 2 \mathrm{LiX}(\sim 0.5 \mathrm{M}$ in THF, full conversion) was determined by titration against $\mathrm{I}_{2}$ (Knochel's method). ${ }^{17}$ The homogeneous $\mathrm{R}_{3} \mathrm{SiMgX} \cdot 2 \mathrm{LiX}$ solution could be stored in a Schlenk flask purged with $\mathrm{N}_{2}$ at $2-8{ }^{\circ} \mathrm{C}$ in a fridge.

The color of the $\mathrm{R}_{3} \mathrm{SiMgX} \cdot 2 \mathrm{LiX}$ solution depends on the substitution at the silicon atom: $\mathrm{Me}_{2} \mathrm{PhSiMgX} \cdot 2 \mathrm{LiX} 1 \mathrm{a}$ (purple), $\mathrm{MePh}_{2} \mathrm{SiMgX} \cdot 2 \mathrm{LiX}$ 1b (light purple), $\mathrm{Ph}_{3} \mathrm{SiMgX} \cdot 2 \mathrm{LiX}$ 1c (brown), $t$ - $\mathrm{BuPh}_{2} \mathrm{SiMgX} \cdot 2 \mathrm{LiX}$ 1d (light green), $t$-Bu(Me)PhSiMgX.2LiX $1 e$ (light purple), $\left(\mathrm{Et}_{2} \mathrm{~N}\right) \mathrm{Ph}_{2} \mathrm{SiMg}-$ $\mathrm{Br} \cdot 2 \mathrm{LiX} \mathbf{1 f}$ (gray).

\section{Copper-Catalyzed Allylic Substitution with $\mathrm{R}_{3} \operatorname{SiMgX} 1$; General Procedure 2 (GP 2)}

A flame-dried Schlenk flask equipped with a stir bar was charged with $\mathrm{CuI}(1.9 \mathrm{mg}, 0.010 \mathrm{mmol}, 2.0 \mathrm{~mol} \%)$. The flask was evacuated and backfilled with $\mathrm{N}_{2}(3 \times)$ followed by the addition of THF $(1 \mathrm{~mL})$. After stirring for $10 \mathrm{~min}$ at r.t., the indicated allylic precursor $(0.50 \mathrm{mmol}$, 1.0 equiv) was added, and the solution was brought to $0{ }^{\circ} \mathrm{C}$. Then, the corresponding $\mathrm{R}_{3} \mathrm{SiMgX} 1$ ( $0.60 \mathrm{mmol}, 1.2$ equiv) was added over 1 min. After $1 \mathrm{~h}$, the reaction was quenched with sat. aq $\mathrm{NH}_{4} \mathrm{Cl}(5 \mathrm{~mL})$. $\mathrm{CH}_{2} \mathrm{Cl}_{2}(20 \mathrm{~mL})$ was added for extraction, and the $\mathrm{CH}_{2} \mathrm{Cl}_{2}$ layer was washed with brine $(20 \mathrm{~mL})$ and $\mathrm{H}_{2} \mathrm{O}(20 \mathrm{~mL})$. The aqueous phase was extracted with $\mathrm{CH}_{2} \mathrm{Cl}_{2}(2 \times 20 \mathrm{~mL})$. The combined organic phases were dried (anhyd $\mathrm{Na}_{2} \mathrm{SO}_{4}$ ), filtered, and the solvents were evaporated under reduced pressure. Purification of the residue by flash column chromatography on silica gel with indicated solvent as eluent afforded the silylated product.

\section{(E)-Cinnamyldimethyl(phenyl)silane [ $\alpha-(E)-3 a]$}

Prepared from (E)-cinnamyl acetate $[(E)-\mathbf{2 a} ; 88 \mathrm{mg}, 0.50 \mathrm{mmol}] \mathrm{ac}-$ cording to GP 2 with $\mathrm{Me}_{2} \mathrm{PhSiMgX} 1 \mathrm{a}$ at $0{ }^{\circ} \mathrm{C}$. Purification by flash column chromatography on silica gel using $n$-pentane afforded $\alpha-(E)$-3a as a colorless oil; yield: $120 \mathrm{mg}$ (95\%, contaminated with 1,1,2,2-tetramethyl-1,2-diphenyldisilane); $R_{f}=0.60$ ( $n$-pentane).

${ }^{1} \mathrm{H} \mathrm{NMR}\left(500 \mathrm{MHz}, \mathrm{CDCl}_{3}\right): \delta=0.31(\mathrm{~s}, 6 \mathrm{H}), 1.90(\mathrm{~d}, J=6.5 \mathrm{~Hz}, 2 \mathrm{H})$, 6.16-6.26 (m, 2 H), 7.12-7.17 (m, $1 \mathrm{H}$ ), 7.24-7.26 (m, $4 \mathrm{H}$ ), 7.35-7.38 ( $\mathrm{m}, 3 \mathrm{H}), 7.49-7.55(\mathrm{~m}, 2 \mathrm{H})$.

${ }^{13} \mathrm{C}$ NMR $\left(125 \mathrm{MHz}, \mathrm{CDCl}_{3}\right): \delta=-3.3,23.0,125.6,126.3,127.1,127.8$, $128.4,128.9,129.1,133.6,138.4,138.5$.

${ }^{29} \mathrm{Si}$ DEPT NMR (99 MHz, $\left.\mathrm{CDCl}_{3}\right): \delta=-4.1$.

HRMS (EI): $m / z$ [M] ${ }^{+}$calcd for $\mathrm{C}_{17} \mathrm{H}_{20} \mathrm{Si}$ : 252.1334; found: 252.1332 .

The spectroscopic data are in accordance with those reported. ${ }^{4 c}$

\section{(E)-Geranyldimethyl(phenyl)silane [ $\alpha-(E)-8 a]$}

Prepared from (E)-geranyl acetate [(E)-4a; $98 \mathrm{mg}, 0.50 \mathrm{mmol}]$ according to GP 2 with $\mathrm{Me}_{2} \mathrm{PhSiMgX} 1 \mathrm{a}$ at $0{ }^{\circ} \mathrm{C}$. Purification by flash column chromatography on silica gel using $n$-pentane afforded $\alpha-(E)-8 \mathbf{a}$ as a colorless oil; yield: $119 \mathrm{mg}(87 \%) ; R_{f}=0.65$ ( $n$-pentane).

${ }^{1} \mathrm{H}$ NMR $\left(500 \mathrm{MHz}, \mathrm{CDCl}_{3}\right): \delta=0.26(\mathrm{~s}, 6 \mathrm{H}), 1.50(\mathrm{~s}, 3 \mathrm{H}), 1.61(\mathrm{~s}, 3 \mathrm{H})$, $1.64(\mathrm{~d}, J=8.6 \mathrm{~Hz}, 2 \mathrm{H}), 1.69(\mathrm{~s}, 3 \mathrm{H}), 1.97-2.02(\mathrm{~m}, 2 \mathrm{H}), 2.03-2.10(\mathrm{~m}$, $2 \mathrm{H}), 5.09$ (tt, $J=6.7,1.4 \mathrm{~Hz}, 1 \mathrm{H}$ ), 5.17 (tq, $J=8.6,1.4 \mathrm{~Hz}, 1 \mathrm{H}$ ), 7.317.38 (m, $3 \mathrm{H}), 7.49-7.55(\mathrm{~m}, 2 \mathrm{H})$.

${ }^{13} \mathrm{C}$ NMR $\left(125 \mathrm{MHz}, \mathrm{CDCl}_{3}\right): \delta=-3.3,15.8,17.67,17.69,25.7,26.9$, 40.0, 119.6, 124.6, 127.7, 128.8, 131.2, 133.1, 133.6, 139.3 .

${ }^{29} \mathrm{Si}$ DEPT NMR $\left(99 \mathrm{MHz}, \mathrm{CDCl}_{3}\right): \delta=-3.8$.

HRMS (EI): $m / z$ [M] $]^{+}$calcd for $\mathrm{C}_{18} \mathrm{H}_{28} \mathrm{Si}$ : 272.1955; found: 272.1952 .

The spectroscopic data are in accordance with those reported. ${ }^{4 a}$ 


\section{(Z)-Neryldimethyl(phenyl)silane $[\alpha-(Z)-8 a]$}

Prepared from (Z)-neryl acetate [(Z)-4a; $98 \mathrm{mg}, 0.50 \mathrm{mmol}]$ according to GP 2 with $\mathrm{Me}_{2} \mathrm{PhSiMgX} 1 \mathrm{a}$ at $0{ }^{\circ} \mathrm{C}$. Purification by flash column chromatography on silica gel using $n$-pentane afforded $\alpha-(Z)-8 \mathbf{a}$ as a colorless oil; yield: $124 \mathrm{mg}$ (91\%); $R_{f}=0.65$ ( $n$-pentane).

${ }^{1} \mathrm{H} \mathrm{NMR}\left(500 \mathrm{MHz}, \mathrm{CDCl}_{3}\right): \delta=0.26(\mathrm{~s}, 6 \mathrm{H}), 1.60(\mathrm{~s}, 3 \mathrm{H}), 1.65(\mathrm{~d}, J=$ 8.6 Hz, $2 \mathrm{H}), 1.69(\mathrm{~s}, 6 \mathrm{H}), 1.94-2.02(\mathrm{~m}, 4 \mathrm{H}), 5.07-5.13(\mathrm{~m}, 1 \mathrm{H}), 5.17$ $(\mathrm{t}, J=8.6 \mathrm{~Hz}, 1 \mathrm{H}), 7.33-7.38(\mathrm{~m}, 3 \mathrm{H}), 7.49-7.54(\mathrm{~m}, 2 \mathrm{H})$.

${ }^{13} \mathrm{C}$ NMR $\left(125 \mathrm{MHz}, \mathrm{CDCl}_{3}\right): \delta=-3.2,17.3,17.6,23.4,25.7,26.4,31.7$, $119.7,124.6,127.7,128.8,131.4,133.6,133.9,139.3$.

${ }^{29} \mathrm{Si}$ DEPT NMR (99 MHz, $\left.\mathrm{CDCl}_{3}\right): \delta=-4.2$.

HRMS (EI): $m / z$ [M] ${ }^{+}$calcd for $\mathrm{C}_{18} \mathrm{H}_{28} \mathrm{Si}$ : 272.1955; found: 272.1952 .

The spectroscopic data are in accordance with those reported. ${ }^{4 a}$

\section{(3-Cyclohexylallyl)dimethyl(phenyl)silane (9a)}

Prepared from (E)-(3-bromoprop-1-en-1-yl)cyclohexane [(E)-5h; 102 $\mathrm{mg}, 0.50 \mathrm{mmol}$ ] according to GP 2 with $\mathrm{Me}_{2} \mathrm{PhSiMgX} 1 \mathrm{a}$ at $0{ }^{\circ} \mathrm{C}$. Purification by flash column chromatography on silica gel using $n$-pentane afforded 9a as a colorless oil; yield: $116 \mathrm{mg}$ (90\%, mixture of all isomers). The ratio of different isomers was confirmed by ${ }^{1} \mathrm{H}$ NMR analysis.

$\alpha-(E)-9 a$

$R_{f}=0.70$ (n-pentane).

${ }^{1} \mathrm{H} \mathrm{NMR}\left(500 \mathrm{MHz}, \mathrm{CDCl}_{3}\right): \delta=0.26(\mathrm{~s}, 6 \mathrm{H}), 1.03-1.25(\mathrm{~m}, 5 \mathrm{H}), 1.61-$ $1.71(\mathrm{~m}, 8 \mathrm{H}), 5.19-5.25(\mathrm{~m}, 1 \mathrm{H}), 5.29-5.38(\mathrm{~m}, 1 \mathrm{H}), 7.33-7.37$ (m, 3 $\mathrm{H}), 7.49-7.54$ (m, $2 \mathrm{H})$.

${ }^{13} \mathrm{C} \mathrm{NMR}\left(125 \mathrm{MHz}, \mathrm{CDCl}_{3}\right): \delta=-3.4,21.6,26.1,26.2,33.5,41.0,122.7$, $127.6,128.8,133.7,136.0,139.1$.

${ }^{29} \mathrm{Si}$ DEPT NMR (99 $\left.\mathrm{MHz}, \mathrm{CDCl}_{3}\right): \delta=-4.7$.

HRMS (EI): $m / z[M]^{+}$calcd for $\mathrm{C}_{17} \mathrm{H}_{26} \mathrm{Si}$ : 258.1798; found: 258.1786.

\section{Prenyldimethy(phenyl)silane ( $\alpha-10 a)$}

Prepared from prenyl acetate (6a; $64 \mathrm{mg}, 0.50 \mathrm{mmol}$ ) according to GP 2 with $\mathrm{Me}_{2} \mathrm{PhSiMgX} 1 \mathrm{a}$ at $0{ }^{\circ} \mathrm{C}$. Purification by flash column chromatography on silica gel using $n$-pentane afforded $\alpha \mathbf{- 1 0 a}$ as a colorless oil; yield: $97 \mathrm{mg}$ (95\%); $R_{f}=0.70$ ( $n$-pentane).

${ }^{1} \mathrm{H} \mathrm{NMR}\left(500 \mathrm{MHz}, \mathrm{CDCl}_{3}\right): \delta=0.26(\mathrm{~s}, 6 \mathrm{H}), 1.50(\mathrm{~s}, 3 \mathrm{H}), 1.63(\mathrm{~d}, J=$ $8.6 \mathrm{~Hz}, 2 \mathrm{H}), 1.69(\mathrm{~s}, 3 \mathrm{H}), 5.16(\mathrm{tt}, J=8.6,1.4 \mathrm{~Hz}, 1 \mathrm{H}), 7.31-7.38(\mathrm{~m}, 3$ H), 7.49-7.55 ( $\mathrm{m}, 2 \mathrm{H})$.

${ }^{13} \mathrm{C}$ NMR $\left(125 \mathrm{MHz}, \mathrm{CDCl}_{3}\right): \delta=-3.2,17.6,17.7,25.7,119.3,127.6$, $128.8,129.5,133.6,139.3$.

${ }^{29} \mathrm{Si}$ DEPT NMR $\left(99 \mathrm{MHz}, \mathrm{CDCl}_{3}\right): \delta=-3.8$.

HRMS (EI): $m / z$ [M] $]^{+}$calcd for $\mathrm{C}_{13} \mathrm{H}_{20} \mathrm{Si}$ : 204.1329; found: 204.1329 .

The spectroscopic data are in accordance with those reported. ${ }^{9}$

\section{Dimethyl(2-methylallyl)(phenyl)silane ( $\alpha$-11a)}

Prepared from 3-bromo-2-methylpropene (7h; $68 \mathrm{mg}, 0.50 \mathrm{mmol}$ ) according to GP 2 with $\mathrm{Me}_{2} \mathrm{PhSiMgX} 1 \mathrm{a}$ at $0{ }^{\circ} \mathrm{C}$. Purification by flash column chromatography on silica gel using $n$-pentane afforded $\alpha$-11a as a colorless oil; yield: $82 \mathrm{mg}(86 \%) ; R_{f}=0.70$ ( $n$-pentane).

${ }^{1} \mathrm{H} \mathrm{NMR}\left(500 \mathrm{MHz}, \mathrm{CDCl}_{3}\right): \delta=0.32(\mathrm{~s}, 6 \mathrm{H}), 1.62(\mathrm{~s}, 3 \mathrm{H}), 1.78(\mathrm{~s}, 2 \mathrm{H})$, 4.47-4.50 (m, $1 \mathrm{H}), 4.59-4.62$ ( $\mathrm{m}, 1 \mathrm{H}), 7.32-7.39$ (m, $3 \mathrm{H}), 7.50-7.57$ $(\mathrm{m}, 2 \mathrm{H})$.

${ }^{13} \mathrm{C}$ NMR $\left(125 \mathrm{MHz}, \mathrm{CDCl}_{3}\right): \delta=-2.9,25.2,25.7,108.8,127.7,128.9$, 133.6, 139.1, 143.3.
${ }^{29} \mathrm{Si}$ DEPT NMR $\left(99 \mathrm{MHz}, \mathrm{CDCl}_{3}\right): \delta=-5.0$. HRMS (EI): $m / z$ [M] $]^{+}$calcd for $\mathrm{C}_{12} \mathrm{H}_{18} \mathrm{Si}$ : 190.1172; found: 190.1164 . The spectroscopic data are in accordance with those reported. ${ }^{18}$

\section{Dimethyl(cyclohex-2-en-1-yl)(phenyl)silane (13a)}

Prepared from 3-bromocyclohexene (12h; $81 \mathrm{mg}, 0.50 \mathrm{mmol}$ ) according to GP 2 with $\mathrm{Me}_{2} \mathrm{PhSiMgX} 1 \mathrm{a}$ at $0{ }^{\circ} \mathrm{C}$. Purification by flash column chromatography on silica gel using $n$-pentane afforded 13a as a colorless oil; yield: $91 \mathrm{mg}$ (84\%); $R_{f}=0.75$ ( $n$-pentane).

${ }^{1} \mathrm{H}$ NMR $\left(500 \mathrm{MHz}, \mathrm{CDCl}_{3}\right): \delta=0.28(\mathrm{~s}, 3 \mathrm{H}), 0.29(\mathrm{~s}, 3 \mathrm{H}), 1.43-1.53$ (m, $2 \mathrm{H}), 1.63-1.70(\mathrm{~m}, 1 \mathrm{H}), 1.74-1.82(\mathrm{~m}, 2 \mathrm{H}), 1.88-2.03(\mathrm{~m}, 2 \mathrm{H})$, 5.60-5.69 (m, $2 \mathrm{H}), 7.31-7.39$ ( $\mathrm{m}, 3 \mathrm{H}), 7.50-7.56$ ( $\mathrm{m}, 2 \mathrm{H})$.

${ }^{13} \mathrm{C}$ NMR $\left(125 \mathrm{MHz}, \mathrm{CDCl}_{3}\right): \delta=-4.8\left(\mathrm{SiCH}_{3}\right),-4.6\left(\mathrm{SiCH}_{3}\right), 22.5,23.8$, 25.0, 25.6, 125.9, 127.5, 127.7, 128.9, 133.9, 138.3.

${ }^{29} \mathrm{Si}$ DEPT NMR $\left(99 \mathrm{MHz}, \mathrm{CDCl}_{3}\right): \delta=-2.5$.

HRMS (EI): $m / z$ [M] $]^{+}$calcd for $\mathrm{C}_{14} \mathrm{H}_{20} \mathrm{Si}$ : 216.1334; found: 216.1327.

The spectroscopic data are in accordance with those reported. ${ }^{9}$

\section{Dimethyl(but-2-en-1-yl)(phenyl)silane (15a)}

Prepared from but-3-en-2-yl benzoate (14b; $88 \mathrm{mg}, 0.50 \mathrm{mmol}$ ) according to GP 2 with $\mathrm{Me}_{2} \mathrm{PhSiMgX} 1 \mathrm{a}$ at $0{ }^{\circ} \mathrm{C}$. Purification by flash column chromatography on silica gel using $n$-pentane afforded $\mathbf{1 5 a}$ as a colorless oil; yield: $87 \mathrm{mg}$ (91\%, mixture of all isomers). The ratio of different isomers was confirmed by ${ }^{1} \mathrm{H}$ NMR analysis; $R_{f}=0.70(n$ pentane).

\section{$\gamma-(Z)-15 a$}

${ }^{1} \mathrm{H}$ NMR $\left(500 \mathrm{MHz}, \mathrm{CDCl}_{3}\right): \delta=0.26(\mathrm{~s}, 6 \mathrm{H}), 1.51(\mathrm{~d}, J=6.0 \mathrm{~Hz}, 3 \mathrm{H})$, $1.73(\mathrm{~d}, J=8.6 \mathrm{~Hz}, 2 \mathrm{H}), 5.34-5.43(\mathrm{~m}, 2 \mathrm{H}), 7.33-7.38$ (m, $3 \mathrm{H}), 7.46-$ $7.57(\mathrm{~m}, 2 \mathrm{H})$.

${ }^{29} \mathrm{Si}$ DEPT NMR $\left(99 \mathrm{MHz}, \mathrm{CDCl}_{3}\right): \delta=-3.8$

\section{Y-(E)-15a}

${ }^{1} \mathrm{H} \mathrm{NMR}\left(500 \mathrm{MHz}, \mathrm{CDCl}_{3}\right): \delta=0.29(\mathrm{~s}, 6 \mathrm{H}), 1.61-1.68(\mathrm{~m}, 5 \mathrm{H}), 5.25-$ $5.46(\mathrm{~m}, 2 \mathrm{H}), 7.33-7.38(\mathrm{~m}, 3 \mathrm{H}), 7.46-7.57(\mathrm{~m}, 2 \mathrm{H})$.

${ }^{29} \mathrm{Si}$ DEPT NMR $\left(99 \mathrm{MHz}, \mathrm{CDCl}_{3}\right): \delta=-4.6$.

The spectroscopic data are in accordance with those reported. ${ }^{9}$

\section{anti-Dimethyl(5-methylcyclohex-2-en-1-yl)(phenyl)silane (anti-} 17a)

Prepared from syn-5-methylcyclohex-2-en-1-yl acetate (syn-16a; 77 $\mathrm{mg}, 0.50 \mathrm{mmol}$ ) according to GP 2 with $\mathrm{Me}_{2} \mathrm{PhSiMgX} 1 \mathrm{a}$ at $0{ }^{\circ} \mathrm{C}$. Purification by flash column chromatography on silica gel using $n$-pentane afforded anti-17a as a colorless oil; yield: $109 \mathrm{mg}$ (95\%, mixture of all isomers). The ratio of different isomers was confirmed by ${ }^{1} \mathrm{H}$ NMR analysis; $R_{f}=0.50$ ( $n$-pentane).

${ }^{1} \mathrm{H}$ NMR (500 MHz, CDCl $): \delta=0.29(\mathrm{~s}, 3 \mathrm{H}), 0.30$ (s, $3 \mathrm{H}$ ), 0.88 (d, $J=$ $6.4 \mathrm{~Hz}, 3 \mathrm{H}), 1.41-1.48(\mathrm{~m}, 1 \mathrm{H}), 1.58-1.72(\mathrm{~m}, 3 \mathrm{H}), 1.82-1.87(\mathrm{~m}, 1$ H), 2.01-2.07 (m, $1 \mathrm{H}), 5.55-5.59(\mathrm{~m}, 1 \mathrm{H}), 5.61-5.65(\mathrm{~m}, 1 \mathrm{H}), 7.33-$ 7.37 (m, $3 \mathrm{H})$, 7.49-7.54 (m, $2 \mathrm{H})$.

${ }^{13} \mathrm{C}$ NMR $\left(125 \mathrm{MHz}, \mathrm{CDCl}_{3}\right): \delta=-4.13\left(\mathrm{SiCH}_{3}\right),-4.08\left(\mathrm{SiCH}_{3}\right), 21.2$, $24.9,26.2,31.2,33.0,124.3,127.2,127.7,128.9,133.9,138.5$.

${ }^{29} \mathrm{Si}$ DEPT NMR (99 MHz, $\left.\mathrm{CDCl}_{3}\right): \delta=-2.6$.

HRMS (EI): $m / z$ [M] $]^{+}$calcd for $\mathrm{C}_{15} \mathrm{H}_{22} \mathrm{Si}$ : 230.1491; found: 230.1492 .

The spectroscopic data are in accordance with those reported. ${ }^{3 \mathrm{f}}$ 


\section{Allyldimethyl(phenyl)silane (19a)}

Prepared from allyl methyl carbonate $(\mathbf{1 8 c} ; 58 \mathrm{mg}, 0.50 \mathrm{mmol}) \mathrm{ac}-$ cording to GP 2 with $\mathrm{Me}_{2} \mathrm{PhSiMgX} \mathbf{1 a}$ at $0{ }^{\circ} \mathrm{C}$. Purification by flash column chromatography on silica gel using $n$-pentane afforded 19a as a colorless oil; yield: $84 \mathrm{mg}$ (95\%); $R_{f}=0.65$ ( $n$-pentane).

${ }^{1} \mathrm{H} \mathrm{NMR}\left(500 \mathrm{MHz}, \mathrm{CDCl}_{3}\right): \delta=0.29(\mathrm{~s}, 6 \mathrm{H}), 1.76(\mathrm{~d}, J=8.6 \mathrm{~Hz}, 2 \mathrm{H})$, 4.82-4.92 (m, 2 H), 5.73-5.83 (m, 1 H), 7.33-7.39 (m, 3 H), 7.49-7.55 $(\mathrm{m}, 2 \mathrm{H})$.

${ }^{13} \mathrm{C}$ NMR $\left(125 \mathrm{MHz}, \mathrm{CDCl}_{3}\right): \delta=-3.5,23.7,113.4,127.7,129.0,133.6$, 134.6, 138.7

${ }^{29} \mathrm{Si}$ DEPT NMR $\left(99 \mathrm{MHz}, \mathrm{CDCl}_{3}\right): \delta=-4.7$.

HRMS (EI): $m / z[M]^{+}$calcd for $\mathrm{C}_{11} \mathrm{H}_{16} \mathrm{Si}: 176.1021$; found: 176.1018 .

The spectroscopic data are in accordance with those reported. ${ }^{18}$

\section{Allyl(methyl)diphenylsilane (19b)}

Prepared from allyl methyl carbonate $(\mathbf{1 8 c} ; 58 \mathrm{mg}, 0.50 \mathrm{mmol})$ according to GP 2 with $\mathrm{MePh}_{2} \mathrm{SiMgX} \mathbf{1 b}$ at $0{ }^{\circ} \mathrm{C}$. Purification by flash column chromatography on silica gel using $n$-pentane afforded $\mathbf{1 9 b}$ as a colorless oil; yield: $108 \mathrm{mg}(91 \%) ; R_{f}=0.55$ (n-pentane).

${ }^{1} \mathrm{H}$ NMR (500 MHz, CDCl $): \delta=0.56(\mathrm{~s}, 3 \mathrm{H}), 2.08(\mathrm{~d}, J=8.6 \mathrm{~Hz}, 2 \mathrm{H}$ ), 4.85-4.95 (m, 2 H), 5.75-5.85 (m, 1 H), 7.33-7.40 (m, 6 H), 7.51-7.56 $(\mathrm{m}, 4 \mathrm{H})$.

${ }^{13} \mathrm{C}$ NMR $\left(125 \mathrm{MHz}, \mathrm{CDCl}_{3}\right): \delta=-4.8,22.1,114.2,127.8,129.2,134.1$, $134.5,136.6$.

${ }^{29} \mathrm{Si}$ DEPT NMR (99 MHz, $\left.\mathrm{CDCl}_{3}\right): \delta=-9.6$.

HRMS (EI): $m / z[M]^{+}$calcd for $\mathrm{C}_{16} \mathrm{H}_{18} \mathrm{Si}$ : 238.1178; found: 238.1172 .

The spectroscopic data are in accordance with those reported. ${ }^{19}$

\section{Allyltriphenylsilane (19c)}

Prepared from allyl methyl carbonate $(\mathbf{1 8 c} ; 58 \mathrm{mg}, 0.50 \mathrm{mmol}) \mathrm{ac}-$ cording to GP 2 with $\mathrm{Ph}_{3} \mathrm{SiMgX} 1 \mathrm{c}$ at $0{ }^{\circ} \mathrm{C}$. Purification by flash column chromatography on silica gel using $n$-pentane afforded $19 \mathrm{c}$ as a white solid; yield: $134 \mathrm{mg}$ (89\%); $\mathrm{mp} 90.0-90.8^{\circ} \mathrm{C} ; R_{f}=0.35$ (n-pentane).

${ }^{1} \mathrm{H} \mathrm{NMR}\left(500 \mathrm{MHz}, \mathrm{CDCl}_{3}\right): \delta=2.40(\mathrm{~d}, J=7.8 \mathrm{~Hz}, 2 \mathrm{H}), 4.87-4.98(\mathrm{~m}, 2$ H), 5.81-5.92 (m, $1 \mathrm{H}), 7.33-7.44(\mathrm{~m}, 9 \mathrm{H}), 7.50-7.55$ (m, $6 \mathrm{H})$.

${ }^{13} \mathrm{C}$ NMR $\left(125 \mathrm{MHz}, \mathrm{CDCl}_{3}\right): \delta=21.8,115.1,127.8,129.5,133.8,134.6$, 135.7.

${ }^{29} \mathrm{Si}$ DEPT NMR (99 MHz, $\left.\mathrm{CDCl}_{3}\right): \delta=-13.8$.

HRMS (EI): $m / z$ [M] $]^{+}$calcd for $\mathrm{C}_{21} \mathrm{H}_{20} \mathrm{Si}$ : 300.1334; found: 300.1330 .

The spectroscopic data are in accordance with those reported. ${ }^{19}$

\section{Allyl(tert-butyl)diphenylsilane (19d)}

Prepared from allyl methyl carbonate $(18 c ; 58 \mathrm{mg}, 0.50 \mathrm{mmol})$ according to GP 2 with $t$ - $\mathrm{BuPh}_{2} \mathrm{SiMgX} \mathbf{1 d}$ at $0{ }^{\circ} \mathrm{C}$. Purification by flash column chromatography on silica gel using $n$-pentane afforded $19 d$ as a colorless oil; yield: $115 \mathrm{mg}(82 \%) ; R_{f}=0.65$ ( $n$-pentane).

${ }^{1} \mathrm{H}$ NMR $\left(500 \mathrm{MHz}, \mathrm{CDCl}_{3}\right): \delta=1.07(\mathrm{~s}, 9 \mathrm{H}), 2.08(\mathrm{dt}, J=8.6,1.4 \mathrm{~Hz}, 2$ H), 4.78-4.82 (m, $1 \mathrm{H}), 4.88-4.93(\mathrm{~m}, 1 \mathrm{H}), 5.71-5.82(\mathrm{~m}, 1 \mathrm{H}), 7.33-$ $7.41(\mathrm{~m}, 6 \mathrm{H}), 7.59-7.64(\mathrm{~m}, 4 \mathrm{H})$.

${ }^{13} \mathrm{C}$ NMR $\left(125 \mathrm{MHz}, \mathrm{CDCl}_{3}\right): \delta=18.5,18.8,27.9,114.5,127.5,129.1$, 134.4, 134.7, 136.0 .

${ }^{29} \mathrm{Si}$ DEPT NMR $\left(99 \mathrm{MHz}, \mathrm{CDCl}_{3}\right): \delta=-5.2$.

HRMS (EI): $m / z$ [M] $]^{+}$calcd for $\mathrm{C}_{19} \mathrm{H}_{24} \mathrm{Si}$ : 280.1642; found: 280.1636 .

The spectroscopic data are in accordance with those reported. ${ }^{20}$

\section{Allyl(tert-butyl)(methyl)(phenyl)silane (19e)}

Prepared from allyl methyl carbonate $(\mathbf{1 8 c} ; 58 \mathrm{mg}, 0.50 \mathrm{mmol}) \mathrm{ac}-$ cording to GP 2 with $t$-Bu(Me)PhSiMgX $1 \mathrm{e}$ at $0{ }^{\circ} \mathrm{C}$. Purification by flash column chromatography on silica gel using $n$-pentane afforded 19e as a colorless oil; yield: $99 \mathrm{mg}(91 \%) ; R_{f}=0.65$ ( $n$-pentane).

HPLC-analysis: OJ-RH (Dacial), $\mathrm{MeCN} / \mathrm{H}_{2} \mathrm{O}=65: 35,0.2 \mathrm{~mL} / \mathrm{min}, \lambda=$ $210 \mathrm{~nm}, t_{\mathrm{R}}=47.9,51.1 \mathrm{~min}$.

${ }^{1} \mathrm{H}$ NMR (500 MHz, $\left.\mathrm{CDCl}_{3}\right): \delta=0.28(\mathrm{~s}, 3 \mathrm{H}), 0.90(\mathrm{~s}, 9 \mathrm{H}), 1.81-1.87$ $(\mathrm{m}, 1 \mathrm{H}), 1.93-1.99(\mathrm{~m}, 1 \mathrm{H}), 4.78-4.82(\mathrm{~m}, 1 \mathrm{H}), 4.86-4.92(\mathrm{~m}, 1 \mathrm{H})$, 5.71-5.82 (m, $1 \mathrm{H}), 7.31-7.44(\mathrm{~m}, 3 \mathrm{H}), 7.48-7.55(\mathrm{~m}, 2 \mathrm{H})$.

${ }^{13} \mathrm{C}$ NMR $\left(125 \mathrm{MHz}, \mathrm{CDCl}_{3}\right): \delta=-8.6,17.4,18.7,26.8,113.6,127.5$, 128.9, 134.7, 135.0, 136.0.

${ }^{29} \mathrm{Si}$ DEPT NMR (99 MHz, $\left.\mathrm{CDCl}_{3}\right): \delta=1.5$.

HRMS (EI): $m / z$ [M] $]^{+}$calcd for $\mathrm{C}_{14} \mathrm{H}_{22} \mathrm{Si}$ : 218.1485; found: 218.1482 .

\section{Allyl(ethoxy)diphenylsilane (19f)}

Prepared from allyl methyl carbonate $(\mathbf{1 8 c} ; 58 \mathrm{mg}, 0.50 \mathrm{mmol})$ according to GP 2 with $\left(\mathrm{Et}_{2} \mathrm{~N}\right) \mathrm{Ph}_{2} \mathrm{SiMgX}$ 1f at $0{ }^{\circ} \mathrm{C}$. Afterwards, anhyd EtOH ( $1 \mathrm{~mL})$ and $\mathrm{NH}_{4} \mathrm{Cl}(55 \mathrm{mg}, 2.0 \mathrm{mmol})$ was added, and the reaction mixture was stirred overnight. Purification by flash column chromatography on silica gel using $n$-pentane afforded $19 f$ as a colorless oil; yield: $115 \mathrm{mg}(86 \%) ; R_{f}=0.30$ ( $n$-pentane).

${ }^{1} \mathrm{H}$ NMR $\left(500 \mathrm{MHz}, \mathrm{CDCl}_{3}\right): \delta=1.22(\mathrm{t}, J=7.1 \mathrm{~Hz}, 3 \mathrm{H}), 2.18(\mathrm{dt}, J=7.8$, $1.4 \mathrm{~Hz}, 2 \mathrm{H}), 3.81(\mathrm{q}, J=7.1 \mathrm{~Hz}, 2 \mathrm{H}), 4.88-4.98(\mathrm{~m}, 2 \mathrm{H}), 5.80-5.90(\mathrm{~m}$, $1 \mathrm{H}), 7.35-7.44(\mathrm{~m}, 6 \mathrm{H}), 7.59-7.64(\mathrm{~m}, 4 \mathrm{H})$.

${ }^{13} \mathrm{C}$ NMR $\left(125 \mathrm{MHz}, \mathrm{CDCl}_{3}\right): \delta=18.4,21.9,59.5,115.0,127.8,129.9$, 133.1, 134.70, 134.73 .

${ }^{29} \mathrm{Si}$ DEPT NMR $\left(99 \mathrm{MHz}, \mathrm{CDCl}_{3}\right): \delta=-8.6$.

HRMS (EI): $m / z$ [M $\left.-\mathrm{C}_{3} \mathrm{H}_{5}\right]^{+}$calcd for $\mathrm{C}_{14} \mathrm{H}_{15} \mathrm{OSi}$ : 227.0887; found: 227.0889.

The spectroscopic data are in accordance with those reported. ${ }^{21}$

\section{Funding Information}

This research was supported by the China Scholarship Council (predoctoral fellowship to W.X., 2015-2019) and the Deutsche Forschungsgemeinschaft (Oe 249/15-1). M.O. is indebted to the Einstein Foundation Berlin for an endowed professorship.

\section{Supporting Information}

Supporting information for this article is available online at https://doi.org/10.1055/s-0037-1610309.

\section{References}

(1) For recent reviews, see: (a) Denmark, S. E.; Ambrosi, A. Org. Process Res. Dev. 2015, 19, 982. (b) Yus, M.; González-Gómez, J. C.; Foubelo, F. Chem. Rev. 2013, 113, 5595. (c) Chabaud, L.; James, P.; Landais, Y. Eur. J. Org. Chem. 2004, 15, 3173.

(2) For selected examples with Si-Si compounds, see: (a) Hayashi, T.; Ohno, A.; Lu, S.-j.; Matsumoto, Y.; Fukuyo, E.; Yanagi, K. J. Am. Chem. Soc. 1994, 116, 4221. (b) Moser, R.; Nishikata, T.; Lipshutz, B. H. Org. Lett. 2010, 12, 28. (c) Selander, N.; Paasch, J. R.; Szabó, K. J. J. Am. Chem. Soc. 2011, 133, 409. (d) Larsson, J. M.; Szabó, K. J. J. Am. Chem. Soc. 2013, 135, 443. 
(3) For selected examples with Si-B compounds, see: (a) Vyas, D. J.; Oestreich, M. Angew. Chem. Int. Ed. 2010, 49, 8513. (b) Delvos, L. B.; Vyas, D. J.; Oestreich, M. Angew. Chem. Int. Ed. 2013, 52, 4650. (c) Takeda, M.; Shintani, R.; Hayashi, T. J. Org. Chem. 2013, 78, 5007. (d) Hazra, C. K.; Irran, E.; Oestreich, M. Eur. J. Org. Chem. 2013, 4903. (e) Delvos, L. B.; Hensel, A.; Oestreich, M. Synthesis 2014, 46, 2957. (f) Delvos, L. B.; Oestreich, M. Synthesis 2015, 47, 924.

(4) For selected examples with silicon zinc reagents, see: (a) Oestreich, M.; Auer, G. Adv. Synth. Catal. 2005, 347, 637. (b) Schmidtmann, E. S.; Oestreich, M. Chem. Commun. 2006, 3643. (c) Vyas, D. J.; Oestreich, M. Chem. Commun. 2010, 46, 568. (d) Hensel, A.; Oestreich, M. Chem. Eur. J. 2015, 21, 9062.

(5) For nucleophilic substitution of silicon electrophiles with allylic metal reagents, see: (a) Lennon, P. J.; Mack, D. P.; Thompson, Q. E. Organometallics 1989, 8, 1121. (b) Murakami, K.; Yorimitsu, H.; Oshima, K. J. Org. Chem. 2009, 74, 1415.

(6) Other methods hinge on the silylation of 1,3-dienes or allenes or, more recently, involve formal $\mathrm{C}-\mathrm{H}$ bond silylation: (a) Suginome, M.; Ohmura, T.; Miyake, Y.; Mitani, S.; Ito, Y.; Murakami, M. J. Am. Chem. Soc. 2003, 125, 11174. (b) Larsson, J. M.; Zhao, T. S.; Szabó, K. J. Org. Lett. 2011, 13, 1888. (c) Miller, Z. D.; Li, W.; Belderrain, T. R.; Montgomery, J. J. Am. Chem. Soc. 2013, 135, 15282. (d) MeAtee, J. R.; Yap, G. P. A.; Watson, D. A. J. Am. Chem. Soc. 2014, 136, 10166. (e) Nakai, S.; Matsui, M.; Shimizu, Y.; Adachi, Y.; Obora, Y.J. Org. Chem. 2015, 80, 7317.

(7) Xue, W.; Shishido, R.; Oestreich, M. Angew. Chem. Int. Ed. 2018, 57,12141
(8) George, M. V.; Peterson, D. J.; Gilman, H. J. Am. Chem. Soc. 1960, 82, 403.

(9) Fleming, I.; Higgins, D.; Lawrence, N. J.; Thomas, A. P. J. Chem. Soc., Perkin Trans. 1 1992, 3331.

(10) For the preparation of syn-16a, see: Watson, I. D. G.; Yudin, A. K. J. Am. Chem. Soc. 2005, 127, 17516.

(11) Fleming, I.; Thomas, A. P. J. Chem. Soc., Chem. Commun. 1986, 1456.

(12) Tamao, K.; Kawachi, A.; Ito, K. J. Am. Chem. Soc. 1992, 82, 3989.

(13) For recent reviews on the synthesis of silicon-stereogenic silanes, see: (a) Xu, L.-W. Angew. Chem. Int. Ed. 2012, 51, 12932. (b) Cui, Y.-M.; Lin, Y.; Xu, L.-W. Coord. Chem. Rev. 2017, 330, 37. (c) Shintani, R. Synlett 2018, 29, 388.

(14) Yasui, K.; Fugami, K.; Tanaka, S.; Tamaru, Y. J. Org. Chem. 1995, $60,1365$.

(15) Harris, R. K.; Becker, E. D.; Cabral de Menezes, R.; Goodfellow, S. M.; Granger, P. Pure Appl. Chem. 2001, 73, 1795.

(16) Kofron, W. G.; Baclawski, L. M. J. Org. Chem. 1976, 41, 1879.

(17) Krasovskiy, A.; Knochel, P. Synthesis 2006, 890.

(18) Fleming, I.; Rowley, M.; Cuadrado, P.; González-Nogal, A. M.; Pulido, F. J. Tetrahedron 1989, 45, 413.

(19) Li, Z.; Yang, C.; Zheng, H.; Qiu, H.; Lai, G. J. Organomet. Chem. 2008, 693, 3771.

(20) Barbero, A.; Cuadrado, P.; Gonzalez, A. M.; Pulido, F. J.; Fleming, I. J. Chem. Soc., Perkin Trans. 1 1991, 2811.

(21) Jorapur, Y. R.; Shimada, T. Synlett 2012, 23, 1633. 